\title{
ANÁLISE RETROSPECTIVA DAS UROGRAFIAS EXCRETORAS EM UM SERVIÇO DE RADIOLOGIA DE UM HOSPITAL GERAL*
}

\author{
Marcelo Souto Nacif ${ }^{1}$, Gustavo Federico Jauregui ${ }^{2}$, Alderico Mendonça Neto ${ }^{2}$, Ana Paula \\ Boechat ${ }^{2}$, Ricardo Andrade Fernandes de Mello ${ }^{3}$, Rodrigo Bastos Tostes ${ }^{4}$, Flávio Carvalho Cruz ${ }^{4}$, \\ Alair Augusto Sarmet Moreira Damas dos Santos ${ }^{5}$
}

Resumo OBJETIVO: Fazer uma revisão de 225 exames de urografia excretora, analisando estatisticamente as principais alterações relacionadas com o trato urinário, e reafirmar a utilidade do método. MATERIAIS E MÉTODOS: Estudo retrospectivo e descritivo realizado a partir de levantamento de 225 exames de urografia excretora realizados no período de 2/1/2002 a 29/11/2002, no Serviço de Radiologia do Hospital Santa Cruz da Beneficência Portuguesa de Niterói. RESULTADOS: Do total de 225 exames, 173 (76,9\%) apresentaram alterações radiológicas (anatômicas, congênitas, adquiridas, funcionais, relacionadas a litíase, cirurgias e outros) e $52(23,1 \%)$ foram normais. A alteração mais freqüente foi a urolitíase $(35,9 \%)$, seguida da dilatação do sistema pielocalicial $(9,9 \%)$. Observamos que $110(48,9 \%)$ eram pacientes do sexo masculino e $115(51,1 \%)$, do sexo feminino. 0 paciente mais jovem tinha apenas dois meses de idade e o mais idoso, 84 anos. CONCLUSÃO: Apesar do advento de novas técnicas diagnósticas como o ultra-som, a tomografia computadorizada e a ressonância magnética, a urografia excretora continua tendo importante papel diagnóstico, além de constituir excelente ferramenta de auxílio à programação cirúrgica do urologista. Unitermos: Urografia excretora; Alterações das vias urinárias; Diagnósticos diferenciais.

Abstract Retrospective analysis of intravenous urography examinations in a radiology service of a general hospital. OBJECTIVE: To review 225 intravenous urography examinations in order to statistically analyze the main urinary tract related findings and to revalidate the usefulness of the method. MATERIALS AND METHODS: This is a retrospective and descriptive study based on data of 225 intravenous urography examinations performed between 2/1/2002 and 29/11/2002 at the Department of Radiology of "Hospital Santa Cruz/Beneficência Portuguesa de Niterói", Niterói, RJ, Brazil. RESULTS: In a total of 225 examinations, radiological findings (anatomical, congenital, acquired, functional and others) were seen in $173(76.9 \%)$ whereas 52 (23.1\%) examinations were normal. The most common finding was calculus $(35.9 \%)$, followed by dilatation of the calyceal system (9.9\%). One hundred and ten patients $(48.9 \%)$ were male and $115(51.1 \%)$ were female. The youngest patient was 2-month-old and the oldest was 84-year-old. CONCLUSION: In spite of the new imaging modalities such as ultrasound, computed tomography and magnetic resonance imaging, intravenous urography still remains an important and valuable method for the investigation of the urinary tract. Key words: Intravenous urography; Urinary tract abnormalities; Differential diagnosis.

\section{INTRODUÇÃO}

No início dos estudos urorradiológicos, dispúnhamos somente da radiografia simples do abdome para a avaliação dos rins

* Trabalho realizado no Instituto de Pós-Graduação Médica Carlos Chagas (IPGMCC), Hospital Santa Cruz/Beneficência Portuguesa de Niterói, Niterói, RJ, e na Faculdade de Medicina de Teresópolis, Centro de Ciências Biomédicas da Fundação Educacional Serra dos Órgãos (FMT-CCBM - FESO), Teresópolis, RJ

1. Professor Auxiliar da Disciplina de Radiologia da FMT-CCBM FESO, Pós-graduando do Curso de Especialização em Radiologia do IPGMCC, Mestrando em Radiologia pela Universidade Federal do Rio de Janeiro (UFRJ).

2. Médicos Pós-graduandos do Curso de Especialização em Radiologia do IPGMCC.

3. Especialização em Radiologia pelo IPGMCC, Membro Titular do Colégio Brasileiro de Radiologia e Diagnóstico por Imagem (CBR), Mestre em Radiologia pela UFRJ.

4. Monitores da Disciplina de Radiologia da FMT-CCBM FESO.

5. Professor Titular do Curso de Especialização em Radiologia do IPGMCC, Mestre e Doutor em Radiologia pela UFRJ, Chefe do Serviço de Imagem do Hospital de Clínicas de Niterói e da VOT Imagem, Professor Adjunto e Chefe do Serviço de Radiologia do HUAP-UFF. e da bexiga. Nos anos 20, uma suspensão de prata coloidal (Collargol) foi introduzida, numa tentativa de melhorar o exame dos tratos superiores e da bexiga, através de injeção retrógrada. Outros agentes foram subseqüentemente tentados, incluindo ar, dióxido de carbono e uma variedade de compostos de metais pesados ${ }^{(1,2)}$.

Um estado de euforia foi atingido no início dos anos 30, quando se deu o desenvolvimento do primeiro material de contraste intravenoso relativamente seguro, o Uroselectan $^{\circledR}$, creditado a Moses Swick, um jovem urologista americano. Consistia de um único átomo de iodo ligado a um

Endereço para correspondência: Dr. Marcelo Souto Nacif. Rua Álvares de Azevedo, 130/704, Icaraí. Niterói, RJ, 24220-021. Alvares de Azevedo, 130/704, Icarai. Niterói, RJ, 24220-021.
E-mail: marcelonacif30@hotmail.com/msnacif@yahoo.com.br Recebido para publicação em 27/5/2003. Aceito, após revisão, em 15/12/2003. anel piridínico com cinco carbonos. Compostos diiodo foram prontamente desenvolvidos $^{(3)}$

Com a introdução, nos anos 50 e 60, do iotalamato de meglumina $\left(\right.$ Conray $\left.{ }^{\circledR}\right)$, do diatrizoato de sódio (Hypaque ${ }^{\circledR}$ ) e dos derivados do ácido triiodobenzóico, a urografia excretora (UE) tornou-se o principal método de diagnóstico por imagem urológico. $\mathrm{O}$ aprimoramento das técnicas, com protocolos bem estabelecidos e constantemente atualizados, como a incorporação da nefrotomografia como parte da rotina urográfica (Bosniak, anos 70), o aperfeiçoamento interpretativo por parte dos radiologistas, e a contínua evolução dos meios de contraste intravenosos iodados (contraste não-iônico introduzido na prática radiológica em meados dos anos 70), contribuíram para fazer perdurar esse status ${ }^{(\mathbf{1 , 3 - 5})}$. 
Outras modalidades de imagem foram sendo paralelamente desenvolvidas, como a ultra-sonografia (US), a tomografia computadorizada (TC) e a ressonância magnética (RM), e apesar do seu crescente uso e associações, o exame global ideal do trato urinário ainda é motivo de controvérsia.

Não obstante, a UE segue sendo extremamente importante para a avaliação de diversas alterações urológicas. Ainda constitui o exame mais adequado para a investigação do sistema coletor (ductos coletores, cálices e sistema coletor intra-renal, ureteres).Também é apropriada para a avaliação de anormalidades congênitas, seguimentos cirúrgicos e terapêuticos, e o estudo da função renal. Quando corretamente realizada, pode fornecer detalhes diagnósticos precisos, não atingidos por outras modalidades de imagem ${ }^{(\mathbf{3 - 6})}$.

É, portanto, fundamental, reafirmar a validade e atualidade do método, assim como manter seu aprimoramento técnico e interpretativo.

\section{Objetivos}

Fazer uma revisão de 225 exames de UE, analisando estatisticamente as principais alterações relacionadas com o trato urinário, bem como relacionar o número de exames sem alterações apreciáveis, e suas distribuições por sexo e faixa etária.

Reafirmar, através da observação das diversas alterações encontradas, bem como das hipóteses afastadas, a utilidade e atual validade do método, principalmente em serviços ou localidades onde o acesso a outros métodos de imagem não está disponível.

\section{MATERIAIS E MÉTODOS}

Estudo observacional, retrospectivo e descritivo, realizado no Serviço de Radiologia do Hospital da Beneficência Portuguesa de Niterói (Santa Cruz Scan), onde funciona o Curso de Especialização em Radiologia do Instituto de Pós-Graduação Médica Carlos Chagas (IPGMCC). O trabalho foi realizado a partir do levantamento dos laudos armazenados no banco de dados dos computadores do Santa Cruz Scan. A amostra consistiu do levantamento de 225 exames de UE realizados no período de 2/1/2002 a 29/11/2002, no Ser- viço de Radiologia do Hospital Santa Cruz da Beneficência Portuguesa de Niterói.

\section{RESULTADOS}

Do total de 225 exames realizados neste período, $173(76,9 \%)$ apresentavam alterações radiológicas e $52(23,1 \%)$ exames tiveram o laudo de normais.

Quanto à idade, o paciente mais novo tinha dois meses de idade e o mais velho, 84 anos, sendo a idade média de 42,6 anos. Os dados relativos à faixa etária estão demonstrados na Tabela 1. Observamos que, quanto ao sexo, 110 pacientes $(48,9 \%)$ eram do sexo masculino e 115 pacientes $(51,1 \%)$ eram do sexo feminino.

Do total de 384 diagnósticos e achados radiológicos, observamos que o grande diagnóstico encontrado foi o de cálculo, com $138(35,9 \%)$ dos achados. Estes foram estudados separadamente e estavam distri- buídos da seguinte forma: renal, 73 (52,9\%) (Figura 1); ureteral com dilatação a montante, $56(40,6 \%)$; radiotransparente, 5 (3,6\%); coraliforme, 2 (1,4\%) (Figura 2); intravesical, $1(0,7 \%)$; e na vesícula biliar, $1(0,7 \%)$, entrando como um importante diagnóstico diferencial.

No que se refere à descrição dos diagnósticos e achados radiológicos no geral, observamos, além do estudo da distribuição dos cálculos, também por ordem de freqüência: dilatação do sistema pielocalicial, 38 (9,9\%); flebólitos, 32 (8,3\%); retardo da eliminação renal, 25 (6,5\%); aumento do resíduo pós-miccional, $22(5,7 \%)$; aumento do volume renal, 15 (4,2\%) (Figura 3); duplicidade pielocalicial e ureteral incompleta, 14 (3,6\%) (Figura 4); cisto parapiélico, $12(3,1 \%)$; redução da relação córtico-medular, 9 (2,3\%); cistocele, 7 $(1,8 \%)$; estenose da junção ureterovesical, 7 (1,8\%); compressão extrínseca do ureter

Tabela 1 Faixa etária dos pacientes.

\begin{tabular}{|ll|c|c|}
\hline \multicolumn{2}{|c|}{ Faixa etária } & № de pacientes & Porcentagem \\
\hline - Recém-nato & (0-28 dias) & 0 & $0 \%$ \\
- Lactente & (29 dias-2 anos incompletos) & 1 & $0,44 \%$ \\
- Pré-escolar & (2 anos-6 anos incompletos) & 0 & $0 \%$ \\
- Escolar & (6 anos-12 anos incompletos) & 2 & $0,89 \%$ \\
- Adolescente & (12 anos-18 anos incompletos) & 5 & $2,22 \%$ \\
- Adulto jovem & (18 anos-35 anos) & 83 & $36,89 \%$ \\
- Adulto & (35 anos-65 anos) & 112 & $49,78 \%$ \\
- Idoso & (mais de 65 anos) & 22 & $9,78 \%$ \\
\hline
\end{tabular}

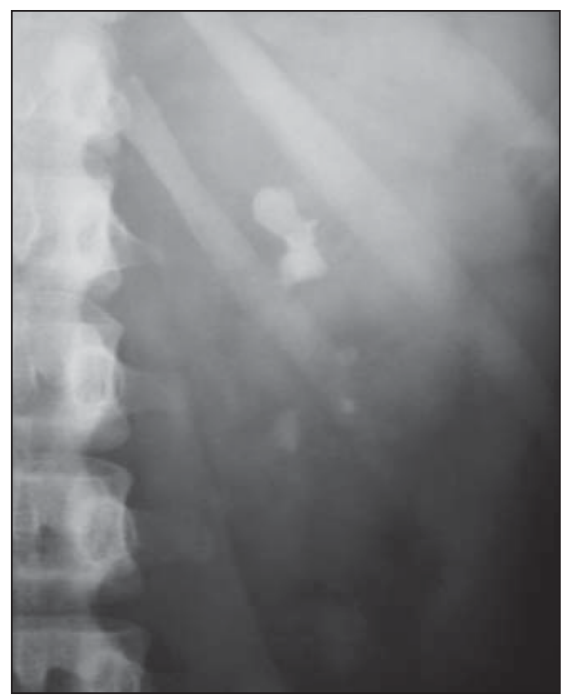

A

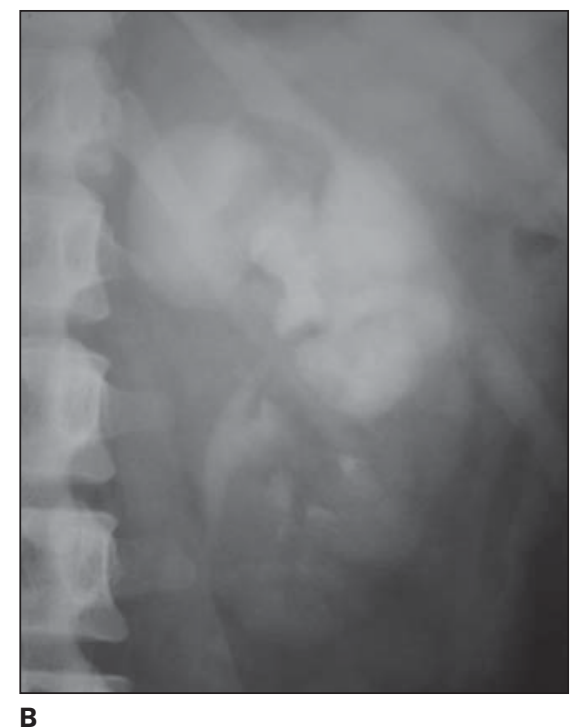

Figura 1. A: Cálculos no rim esquerdo. B: Dilatação do sistema pielocalicial, evidenciada após a injeção do contraste. 


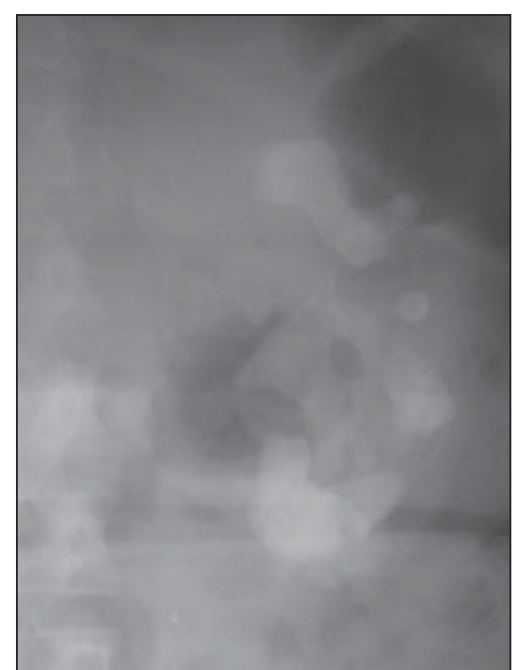

Figura 2. Cálculo coraliforme.

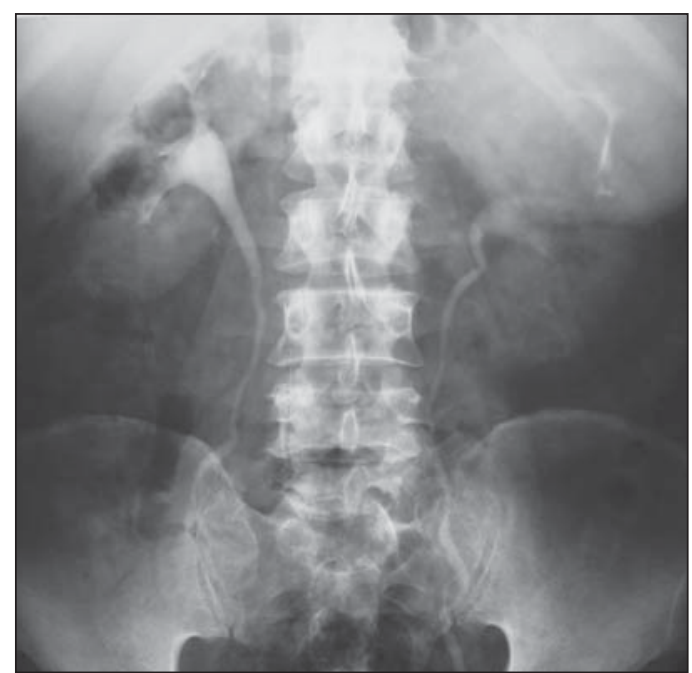

Figura 3. Aumento volumétrico do rim esquerdo (massa).

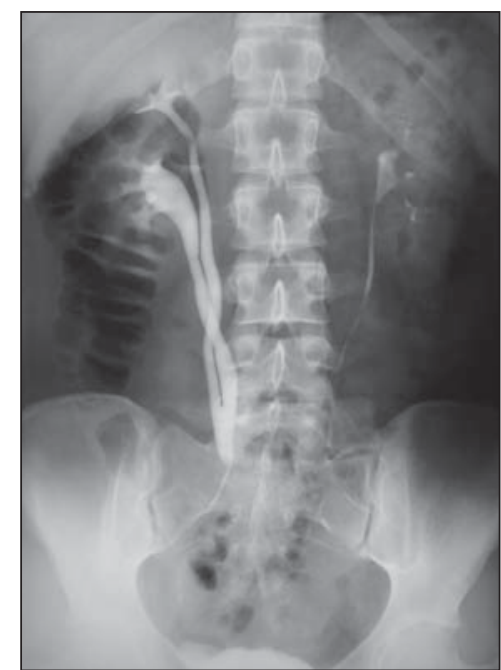

Figura 4. Duplicidade incompleta do sistema pielocalicial e do ureter direito. por mioma, $6(1,7 \%)$; cateter duplo "J", 5 (1,3\%); pelve extra-renal, 5 (1,3\%); cálculo radiotransparente, $5(1,3 \%)$; bexiga de esforço, 5 (1,3\%); redução do tamanho do rim com afilamento da cortical, $5(1,3 \%)$; hipotonia ureteral, 5 (1,3\%); duplicidade pielocalicial completa, $4(1,0 \%)$; e outros, $37(9,6 \%)$.

Devido à baixa freqüência dos achados pertinentes a "outros", associado à importância dos resultados destes diagnósticos quando feitos pela UE, os subdividimos e demonstramos na Tabela 2 (Figuras 5 a 9).

\section{DISCUSSÃO}

A constante evolução dos métodos de diagnóstico por imagem permitiu importantes avanços na investigação e no tratamento das doenças do trato urinário, cada modalidade mostrando benefícios distintos e muitas vezes complementares, e também obstáculos a serem superados, com o objetivo de se atingir o "método ideal".

Especificidades e indicações mais precisas são encontradas para a utilização de cada método. A TC, por exemplo, vem-se tornando o exame de escolha para a avaliação da doença litiásica (principalmente nos grandes centros desenvolvidos), neoplasias renais císticas e sólidas e anormalidades do nefrograma. A US mostra-se efetiva para a avaliação de cistos renais benignos simples e complexos, bem como para o estudo da obstrução em pacientes com alteração da função renal. A RM com

Tabela 2 Descrição dos diagnósticos e achados radiológicos incluídos em "outros".

\begin{tabular}{|l|c|c|}
\hline \multicolumn{1}{|c|}{ Descrição } & № de pacientes & Porcentagem \\
\hline Falhas de enchimento no ureter & 4 & $1,04 \%$ \\
Calcificações prostáticas & 3 & $0,78 \%$ \\
Cisto cortical & 3 & $0,78 \%$ \\
Estenose da junção pieloureteral & 3 & $0,78 \%$ \\
Cálculo coraliforme & 3 & $0,78 \%$ \\
Parada abrupta no ureter (tumor) & 2 & $0,52 \%$ \\
Divertículo de bexiga & 2 & $0,52 \%$ \\
Refluxo vesicoureteral & 2 & $0,52 \%$ \\
Defeito de fusão posterior do arco posterior no sacro & 2 & $0,52 \%$ \\
Compressão extrínseca da bexiga por mioma & 2 & $0,52 \%$ \\
Falha de enchimento na pelve renal & 2 & $0,52 \%$ \\
Baqueteamento dos cálices & 1 & $0,26 \%$ \\
Doença de Paget na bacia & 1 & $0,26 \%$ \\
Rim mal rodado & 1 & $0,26 \%$ \\
Rim pélvico & 1 & $0,26 \%$ \\
Cálculo intravesical & 1 & $0,26 \%$ \\
Cálculo radiopaco de vesícula biliar & 1 & $0,26 \%$ \\
Fístula vesicouterina & 1 & $0,26 \%$ \\
Abscesso (bacilo de Koch) & 1 & $0,26 \%$ \\
Agenesia renal & 1 & $0,26 \%$ \\
\hline
\end{tabular}

contraste tem o seu papel na análise de massas renais indeterminadas e na avaliação de pacientes com riscos significativos de reação adversa ao contraste iodado ${ }^{(1-3)}$.

Da mesma forma, a UE continua tendo suas especificidades e indicações, e mantém, como observado pelo grande número de esclarecimentos diagnósticos constatados por este trabalho, sua atualidade e validade como um método diagnóstico de excelência na investigação das alterações do trato urinário. É primordial manter e aprimorar o refinamento das indicações, para obter sempre a melhor e mais fidedigna informação, além de evitar a realização de exames desnecessários, a exposição excessiva dos pacientes (seja a radiações, a possíveis reações adversas, ou mesmo à ansiedade), e a escalada dos custos investigativos $^{(7,8)}$.

Cerca de 76,9\% dos exames de UE avaliados em nosso trabalho apresentaram 


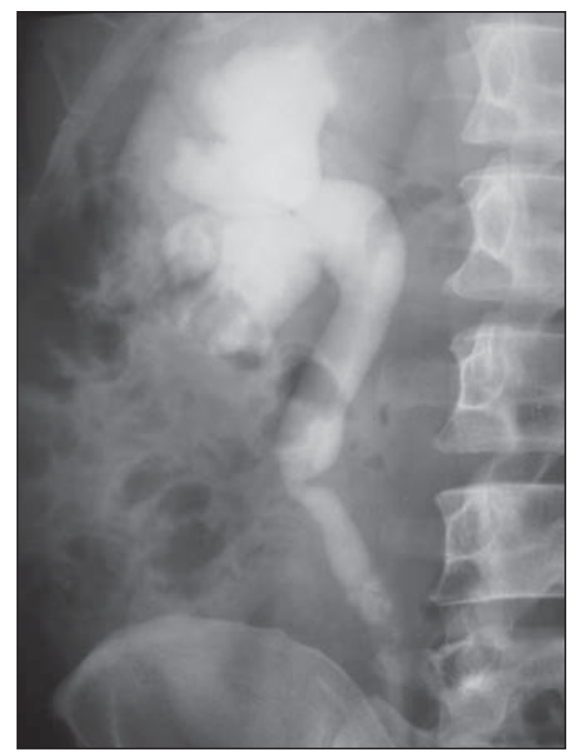

Figura 5. Falha de enchimento no terço médio do ureter direito.

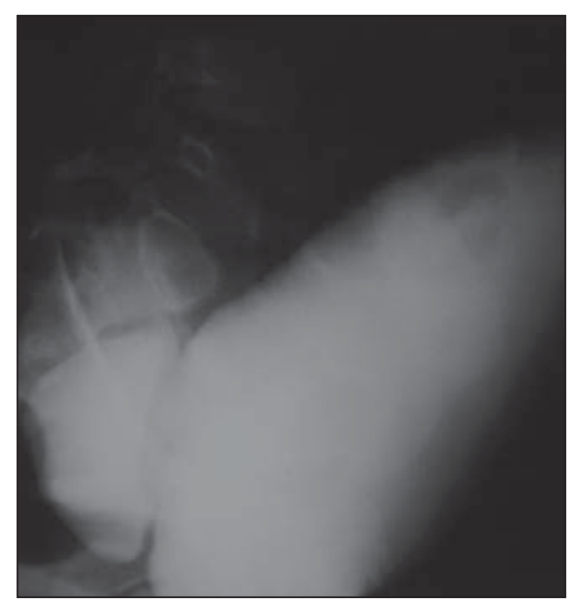

Figura 7. Divertículo vesical.

alterações urológicas, o que vem ressaltar a precisão do método na detecção dessas alterações.

A UE continua sendo a modalidade de imagem mais acurada para a visualização das superfícies uroteliais e avaliação de suas anormalidades potenciais (carcinoma de células transicionais, estriações da mucosa, pielite cística, necrose papilar, etc.). Estas anormalidades responderam por $0,5 \%$ das alterações encontradas (representadas pelo achado de falhas de enchimento calicial e ureteral) $)^{(7,9)}$.

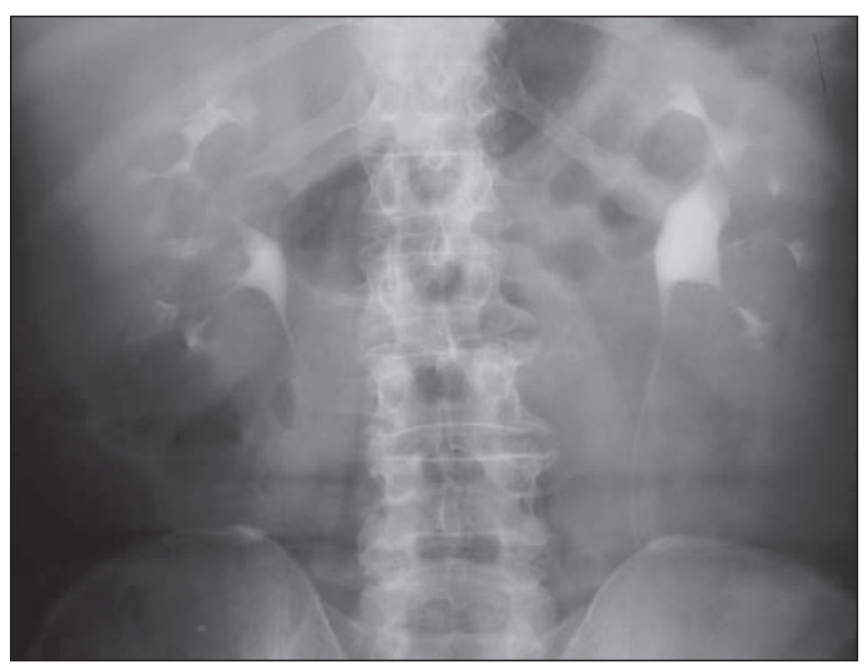

Figura 6. Cistos renais bilaterais.

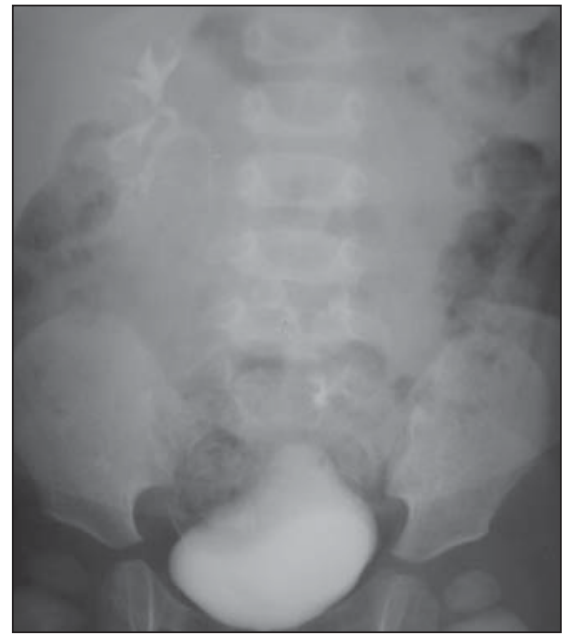

Figura 8. Rim pélvico à esquerda.

A UE é, ainda, um exame apropriado para a avaliação de anormalidades congênitas, fornecendo informações precisas da anatomia alterada ou normal. Duplicidade do sistema pielocalicial e ureteral incompleta representou $3,6 \%$ dos achados, enquanto a duplicidade completa representou $1,0 \%$, constituindo as principais alterações congênitas observadas no nosso trabalho.

O principal achado foi a urolitíase, representando, em grande parte, uma preferência dos urologistas pela investigação da

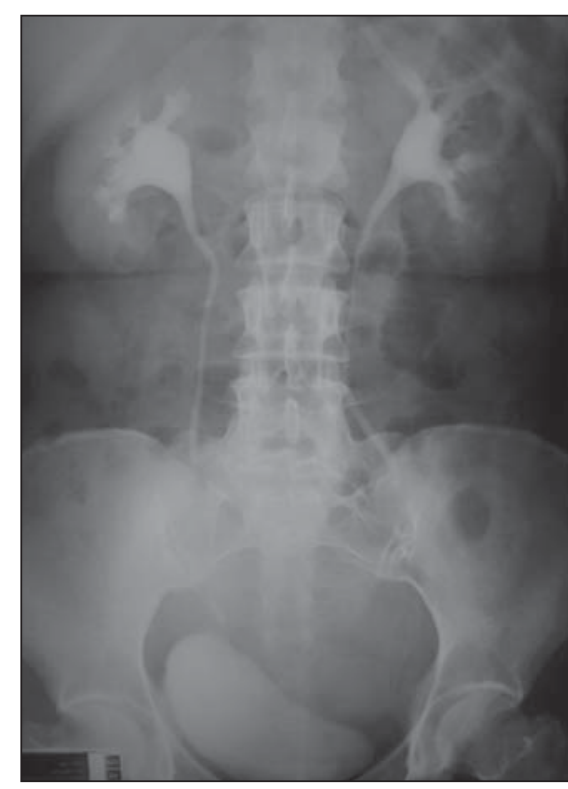

Figura 9. Compressão extrínseca da bexiga e do ureter esquerdo por miomatose uterina.

doença litiásica através do estudo pela UE, refletindo o desconforto que ainda sentem em examinar e tomar decisões baseadas em outros métodos de imagem (como, por exemplo, a TC) $)^{(8,10)}$

A investigação de hematúria freqüentemente se faz pela UE, com avaliação concomitante do parênquima renal e do urotélio. Associada à nefrotomografia, é capaz de identificar cerca de $85 \%$ das lesões iguais ou maiores que $3 \mathrm{~cm}$. A sensibilidade diminui bastante com a redução do tamanho das lesões, exigindo a complementação 
com outros métodos de imagem. Atualmente, tem-se falado muito na introdução da uro-TC e da uro-RM, métodos que consistiriam de uma fusão da TC ou da RM com a UE, que ainda exigem definições de protocolos e estudos que comprovem seus reais papéis diagnósticos ${ }^{(\mathbf{8 , 1 0})}$.

Assim, apesar do advento de novas técnicas diagnósticas como a US, a TC e a RM, a UE continua tendo um importante papel diagnóstico, além de constituir excelente ferramenta de auxílio à programação cirúrgica do urologista.

\section{CONCLUSÃO}

Devemos manter uma atualização continuada no que diz respeito às novas técnicas de imagem, visando à expansão do conhecimento. Não podemos, porém, permitir que isto se faça mediante a subvalorização inconseqüente da radiologia con- vencional, método cuja eficácia não deve ser subestimada. Um estudo urográfico bem orientado permite ótima visualização de porções seqüencialmente opacificadas do trato urinário e provê detalhes diagnósticos não oferecidos pelas outras modalidades de imagem disponíveis. É importante, para isso, compreendermos as limitações inerentes ao método e estimularmos o aprendizado interpretativo contínuo. A capacidade para correlacionar os achados urográficos com os de outros métodos de imagem continua sendo uma importante habilidade a ser aprimorada até o surgimento do método ideal.

\section{REFERÊNCIAS}

1. Amis ES Jr. Epitaph for the urogram (editorial). Radiology 1999;213:639-40.

2. Becker JA, Pollack HM, McClennan BL. Urography survives (letter). Radiology 2001;218:299300

3. Friedenberg RM, Harris RD. Excretory urography in the adult. In: Pollack HM, McClennan BL, Dyer R, Kenney PJ, eds. Clinical urography. 2nd ed. Philadelphia, PA: Saunders, 2000;147-257.

4. Pfister RC, Shea TE. Nephrotomography: performance and interpretation. Radiol Clin North Am 1971;9:41-62.

5. Freitas LO, Nacif MS. Radiologia prática para o estudante de medicina. Rio de Janeiro, RJ: Revinter, 2001.

6. Amis ES Jr. Excretory urography. In: Taveras JM, Ferrucci JT, eds. Radiology: diagnosis-imagingintervention. Philadelphia, PA: Lippincott-Raven, 1997:1-12.

7. Warshauer DM, McCarthy SM, Street L, et al. Detection of renal masses: sensitivities and specificities of excretory urography/linear tomography, US and CT. Radiology 1988;169:363-5.

8. Dyer RB, Chen MYM, Zagoria RJ. Intravenous urography: technique and interpretation. RadioGraphics 2001;21:799-824.

9. Smith RC, Rosenfield AT, Choe KA, et al. Acute flank pain: comparison of non-contrast-enhanced CT and intravenous urography. Radiology 1995; 194:789-94.

10. Goldman SM, Sandler CM. Genitourinary imaging: the past 40 years. Radiology 2000;215:31324 